Original Article

Joumal of Epilepsy Research pISSN 2233-6249 / eISSN 2233-6257

Received April 12, 2018

Revised June 11, 2018

Accepted November 7, 2018

Corresponding author:

A. Bruce Janati, MD

Neurology Center of Fairfax, 3020 Hamake

Ct, Suite 400, Fairfax, VA 22031, USA

Tel. +1-703-876-0800

Fax. +1-703-876-0866

E-mail; classic.gir155@hotmail.com

\title{
Atypical Interictal Epileptiform Discharges in Electroencephalography
}

\author{
A. Bruce Janati, MD ${ }^{1}$, Naif Saad ALGhasab, MD², Maram Youseef Aldaife, MD², \\ Roohi Khan, MD², Imran Khan, MD², Ahmad Abdullah, MD², Aslam Khan, MD², \\ Tarek Abdelwahed, BS ${ }^{2}$ \\ ${ }^{1}$ Neurology Center of Fairfax, Fairfax, VA, USA; ${ }^{2}$ King Khalid Hospital (KKH), Hail, Saudi Arabia
}

Background and Purpose: A great deal of attention has been focused on "typical" interictal epileptiform discharges (IEDs) in the electroencephalography (EEG) literature. However, there is a paucity of data on "atypical" IEDs, namely, positive sharp waves (PSWs), focal triphasic sharp waves and spikes (FTSWs), sharp slow waves (SSWs), bifid spikes, and "notched" delta. In this present study, we sought to address the pathophysiology, characteristics, and diagnostic significance of "atypical" IEDs in clinical neuroscience. Methods: We prospectively reviewed the EEGs of 1,250 patients from a heterogeneous population over a period of 2 years. We also documented demographic, clinical, and neuroimaging data.

Results: Thirty-one patients had PSWs, 26 had FTSWs, 30 had SSWs, 24 had notched delta, and four had bifid spikes in their EEG data. Ninety-six percent of patients with PSWs had epilepsy whereas $100 \%$ of the FTSW and SSW groups had this diagnosis. In the ND group the rate of epilepsy was $95 \%$ and in the bifid spike group 75\%. Accordingly, "atypical" IEDs are potentially epileptogenic patterns with localizing significance, occurring primarily in younger age groups. We also found that a significant number of these patients had congenital central nervous system anomalies.

Conclusions: We conclude that "atypical" IEDs are rare and under-reported EEG patterns that potentially signify focal epileptogenicity. Our data also stresses the significance of neuroimaging in investigating the possibility of an underlying congenital central nervous system anomaly in this population. (2018;8:55-60)

Key words: Interictal, Epileptiform discharge, Electroencephalography

\section{Introduction}

Interictal epileptiform discharges (IEDs) in an electroencephalogram (EEG) are the product of a summation of excitatory post-synaptic potentials (EPSPs) and inhibitory postsynaptic potentials. IED is an electro-clinical term that is typified by sharp waves and spikes, defined as waveforms that are distinct from background activity with their high voltage (at least two-and-a-half times that of the background amplitude). Their duration may vary from less than $70 \mathrm{~ms}$ (spikes) to greater than 70 ms (sharp waves). The polarity of IED in scalp recordings is typically negative, reflecting the action of perpendicularly oriented dipoles in the cortex with negativity near the surface. $^{2-5}$

Generally speaking, IEDs are considered to be a biomarker of epilepsy; however, certain characteristics of sharp waves and spikes may indicate the presence of specific or syndromic anomalies (e.g., West syndrome, Lombroso's syndrome).

Although a great deal of attention has been drawn to the characteristics of typical IEDs, there is a paucity of data on "atypical" IEDs (alEDs) and their pathophysiology, characteristics, and diagnostic significance. Accordingly, we sought to address this topic in a heterogeneous group of patients and to inform clinicians of the significance of IED characteristics, which we believe is often ignored in interpreting epileptiform discharges in EEGs. This study is underpinned by our assertion that attemptting to broaden the existing knowledge of IEDs in clinical neuroscience is the sine qua non in improving the diagnosis, classification, and management of epilepsies.

\section{Methods}

Between January 1, 2013 and January 1, 2015, we prospectively reviewed a total of 1,250 EEGs performed at King Khalid University 
Hospital, a tertiary-care facility and the hospital general hospital in Ha'il, Kingdom of Saudi Arabia. The EEGs were conducted for hospital inpatients and outpatients, patients referred by peripheral hospitals, and residents of the institute with intellectual disabilities. The EEGs were performed according to the guidelines adopted by the American Clinical Neurophysiology Society. ${ }^{6}$ The EEGs were all reviewed and interpreted by a neurologist certified by the American Board of Neurology and the American Board of Clinical Neurophysiology. A second opinion was obtained from an EEG fellow when alEDs were present. Sleep recordings were obtained in the majority of cases, and hyperventilation was performed in cooperative patients in whom no contraindications to this procedure existed. Photic stimulation was universally performed. Seventy percent of the referrals were made for confirmation and classification of seizures, including epileptic seizures. Other reasons for EEG referrals included cranio-cerebral trauma, encephalopathies, cerebrovascular accidents, delirium, dementia, space-occupying lesions, and neurodevelopmental disorders.

For interpretation of IEDs, we used the guidelines adopted by Maulsby. ${ }^{7}$ In addition to typical sharp waves and spikes, we identified five distinct alEDs: positive sharp waves (PSWs), focal triphasic sharp waves and spikes (FTSWs), sharp slow waves (SSWs), bifid spikes, and "notched" delta. We defined PSWs as IEDs with positive polarity (Fig. 1), with a duration of 80 to $200 \mathrm{~ms}$ and an amplitude of 100 to 300 microvolts. We speculate that PSW reflects an augmentation of the deep cortical EPSPs, giving rise to a prominent positive trough, obfuscating the negative component of the sharp wave or spike on the surface EEG. FTSW are IEDs with prominent sinusoidal (dome-shaped) after-coming slow waves (ASW) with negative polarity, configur-

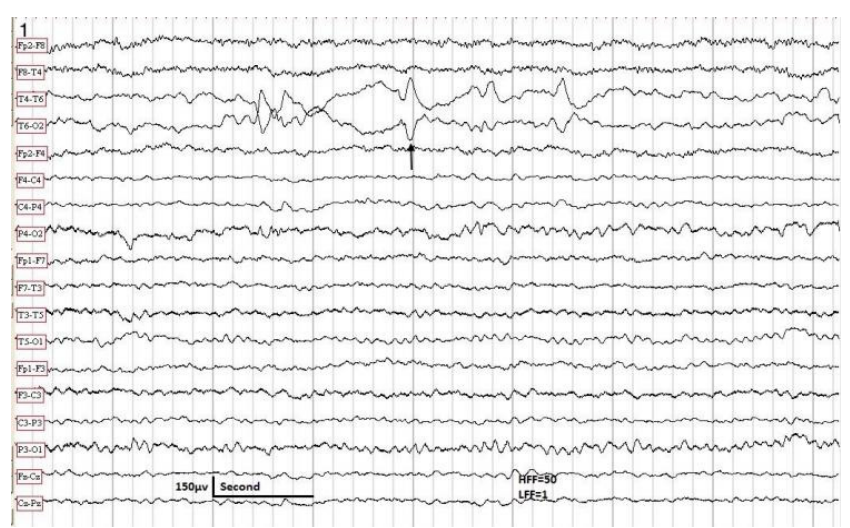

Figure 1. The EEG sample taken in a 10-year-old male with partial epilepsy controlled by carbamazepine. Patient awake. The EEG shows positive sharp waves in the right posterior temporal region (arrow). Neurological examination was normal. No MRI was available. HFF, high frequency filter; LFF, low frequency filter; EEG, electroencephalography; MRI, magnetic resonance imaging. ing a triphasic morphology (Fig. 2). Their amplitude varies from 100 to 300 microvolts with a duration of 150 to $500 \mathrm{~ms}$. The prevailing theory on pathogenesis of this pattern is simultaneous augmentation of EPSPs in both upper and lower cortical neurons (emanating from a local cortical deafferentation), resulting in the generation of a prominent ASW. SSWs are focal, sharply contoured large slow waves with a negative polarity and an amplitude of 150 to 500 microvolts with a duration of 200 to $500 \mathrm{~ms}$ (Fig. 3). The putative theory is that in certain types of cerebral insult there is augmentation of deep cortical EPSPs associated with widespread projection of this activity to the surface, giving rise to SSWs. Bifid spikes are composed of a succession of two negative spikes presenting in a bifid or conjoined morphology resembling the cardiac M pattern in right- bundle- branch block (Fig. 4). The pathogenesis may be a "conduction defect" in projection fibers. Notched delta (ND) is composed of a delta wave superimposed by sharp waves or spikes on its descending or ascending phase (Fig. 5). This pattern is theoretically generated in the cortex where spatiotemporal averaging of electrical signals from deep pacemakers takes place. When these signals reach the cortex asynchronously, they result in the formation of peculiar EEG patterns such as ND. Physiological sharp waves and electrocardiography, environmental, and as well as electrode artifacts were identified and excluded.

Upon completion of the EEG data collection, to collect further pertinent data, we began to retrospectively review the hospital records of patients who had demonstrated alEDs in their EEG. In addition to demographic data the following clinical data were documented: neurological findings, general physical examination, diagnosis, presence or absence of seizures, seizure type, seizure frequency, seizure chron-

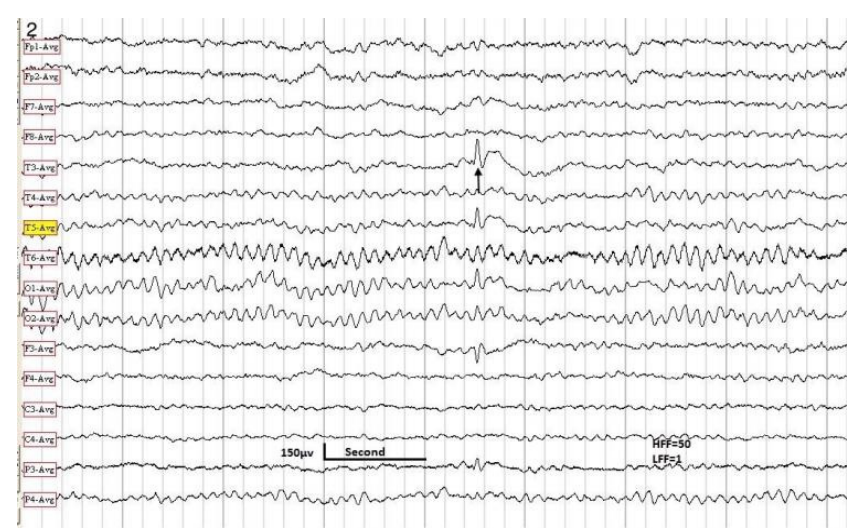

Figure 2. The EEG sample obtained in a 10-year-old male with Lombroso syndrome. Note a triphasic spike in the left mid-temporal region (arrow). HFF, high frequency filter; LFF, low frequency filter; EEG, electroencephalography.

(1)

\section{Copyright (C) 2018 Korean Epilepsy Society}




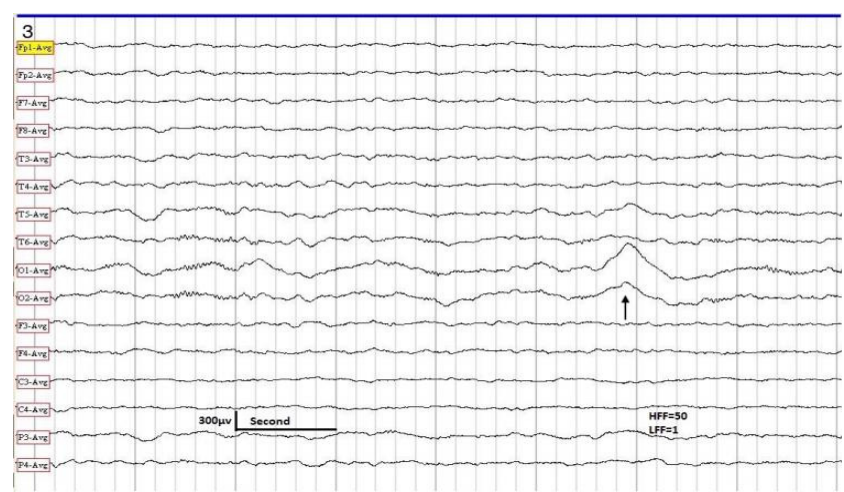

Figure 3. EEG sample taken in a 3-year-old male with generalized convulsive seizures. Patient asleep. The EEG shows SSWs in the left occipital-temporal region (arrow). Normal developmental milestones. MRI of the brain showed agenesis of corpus callosum. HFF, high frequency filter; LFF, low frequency filter; EEG, electroencephalography; SSW, sharp slow wave; MRI, magnetic resonance imaging.

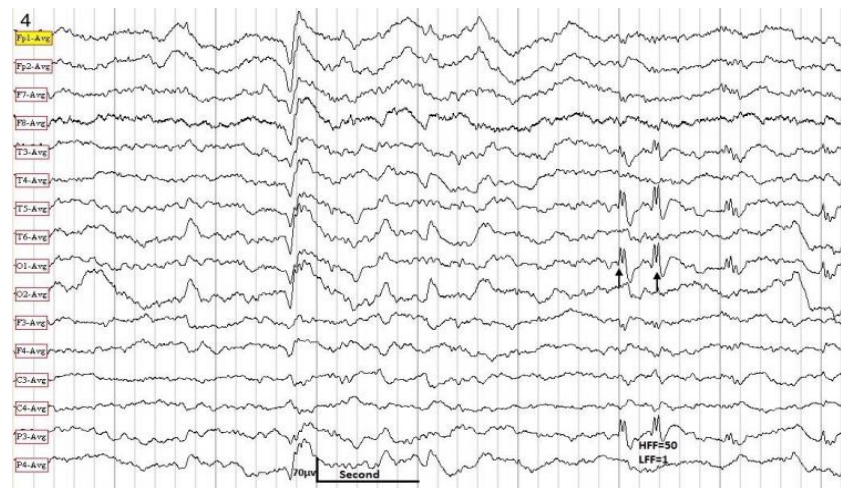

Figure 4. EEG obtained in a 7-year-old male with partial seizures with secondary generalization. The EEG sample shows "bifid" spikes in the left temporaloccipital-parietal region (arrows). Patient was intellectually challenged. There were multiple dysmorphias. HFF, high frequency filter; LFF, low frequency filter; MRI showed an arachnoid cyst and Dandy-Walker syndrome. EEG, electroencephalography; MRI, magnetic resonance imaging.

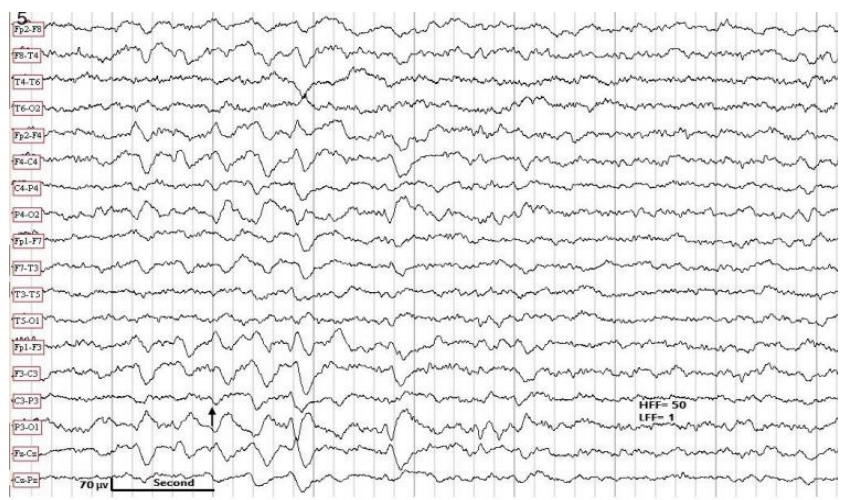

Figure 5. EEG obtained in a 7-year-old female with generalized convulsive seizures. Patient awake. The EEG shows a burst of generalized but posterior-predominant "notched" delta (arrow). Normal MRI. HFF, high frequency filter; LFF, low frequency filter; EEG, electroencephalography; MRI, magnetic resonance imaging. icity, date of last seizure before the EEG (if available), and anticonvulsant regimen. We classified seizures according to the most recent classification system of epilepsies adopted by the ILAE. ${ }^{8}$ Thirty-three patients had focal epilepsy (focal aware, focal impaired awareness, focal non-motor, and focal tonic-clonic). Twelve patients had generalized epilepsy (absence, myoclonic, atonic, tonic, tonic-clonic) and 56 patients had combined focal and generalized seizures. In six patients epilepsy type was unknown whereas two patients had syndromic epilepsy (West syndrome). There was no significant difference of epilepsy types between the group with IEDs only and the group with alED. The following EEG parameters were monitored in all patients: background activity, presence and topography of IEDs, alEDs, and electrographic seizures, focal slowing, and miscellaneous data. The neuroimaging (computed tomography or magnetic resonance imaging [MRI]) data were recorded as follows: presence or absence of atrophy, hydrocephalus, periventricular white matter disease, callosal anomalies, cerebellar or vermian atrophy, brain stem anomalies, white matter abnormal signals, lesions of cortical gray matter or basal ganglia, syndromic congenital anomalies, and miscellaneous findings.

\section{Results}

Of the original 1,250 patients that we reviewed 115 patients had AIEDs in their EEG. There was a preponderance of temporal lobe foci in this population. Age distribution of our patients is shown in Table 1. Thirty patients (2.4\%) had PSWs, 26 patients (2\%) had FTSWs, 30 patients (2.4\%) showed SSWs, 24 (1.9\%) had notched delta, and four $(0.3 \%)$ patients had bifid spikes in their EEG. In one patient PSWs and FTSWs co-existed.

Ninety-six percent of patients with PSWs had a diagnosis of epilepsy, compared to 100\% FTSWs and SSWs patients. Among patients with notched delta 95\% had epileptic seizures and in patients

Table 1. Age distribution of patients with alEDs

\begin{tabular}{lc}
\hline Age, years & $\begin{array}{c}\text { Number of } \\
\text { patients }\end{array}$ \\
\hline $0-10$ & $59(51)$ \\
$11-20$ & $33(29)$ \\
$21-30$ & $16(14)$ \\
$\geq 31$ & $7(6)$ \\
Total & $115(100)$ \\
\hline
\end{tabular}

Values are presented as number (\%).

alED, atypical interictal epileptiform discharge. 
with bifid spikes this percentage was 75\%. Diagnostic categories in this group are summarized in Table 2. Miscellaneous EEG findings are summarized in Table 3. Moreover, 39 patients (34\%) had congenital central nervous system (CNS) anomalies (Table 4). Of the original 1,250 patients that we had reviewed 410 patients had "typical" IEDs in their EEG. In this population we found 25 cases $(6 \%)$ of congenital

Table 2. Neurological disorders in patients with alEDs

\begin{tabular}{lc}
\hline Neurological disorders & $\begin{array}{c}\text { Number of } \\
\text { patients }\end{array}$ \\
\hline Epilepsy & 109 \\
Cerebral palsy & 9 \\
Psychomotor retardation & 4 \\
Hydrocephalus & 7 \\
Syndromic congenital anomalies & 39 \\
\hline
\end{tabular}

Many patients had more than one diagnosis.

alED, atypical interictal epileptiform discharge.

Table 3. Miscellaneous EEG findings in patients with alEDs

\begin{tabular}{lc}
\hline EEG findings & $\begin{array}{c}\text { Number of } \\
\text { patients }\end{array}$ \\
\hline Normal background & 51 \\
Diffuse background slowing & 33 \\
Focal EEG slowing (occasional, intermittent or & 31 \\
continuous; irregular); unilateral or bilaterally & \\
independent. & 12 \\
Generalized epileptiform discharges & 2 \\
Hypsarrythmia & \\
\hline
\end{tabular}

EEG, electroencephalography; alED, atypical interictal epileptiform discharge.
CNS anomalies.

Sixty percent of patients were on monotherapy with successful seizure control, while $40 \%$ of patients required polytherapy. For focal and generalized epilepsy types both old-generation anticonvulsants (carbamazepine, valproic acid, phenytoin, and phenobarbital) and new-generation anticonvulsants (topiramate, lamotrigine, and levetiracetam) were used. Specifically, in genetic generalized epilepsies and in myoclonic epilepsies we used valproic acid in males and lamotrigine in females. Occasionally, we prescribed levetiracetam or topiramate as second line therapy in myoclonic epilepsies. The two patients with West syndrome were treated with a combination of vigabatrin and carbamazepine.

\section{Discussion}

Original studies of IEDs primarily involved intracranial EEGs, therefore marking a milestone in the field of epilepsy. Later studies confirmed that intracranial epileptiform discharges and scalp IEDs have common pathophysiological mechanisms. ${ }^{9}$ Nevertheless, there are some differences: intracranial discharge are more frequent, more variable, shorter in duration, sharper in configuration, and more complex in their topography.

There are several factors that determine the morphology of scalp IEDs. First, the decay time of IEDs depends the dendritic location of synaptic structures and the active and passive membrane properties of the discharging neurons. This defines the "time-constant" (capacitance) of the decay phase. The larger the capacitance of these neurons, the

Table 4. MRI findings in patients with alEDs

\begin{tabular}{|c|c|c|}
\hline Class & Number of patients & Abnormality \\
\hline Normal MRI findings & $37(32)$ & \\
\hline Congenital anomalies & $51(44)$ & $\begin{array}{l}\text { Cerebral atrophy, adrenoleukodystrophy, periventricular calcification and bilateral basal } \\
\text { ganglia calcifications, calcified frontal lobes, enlarged ventricles, partial agenesis of corpus } \\
\text { callosum, absent septum pellucidum, holoprosencephaly, cortical atrophy, periventricular } \\
\text { leukomalacia, corpus callosum anomalies, Dandy-Walker syndrome, Joubert syndrome, } \\
\text { encephalomalacia, cerebellar atrophy, abnormal white matter, capacious cisterna magna } \\
\text { congenital hydrocephalus, cortical atrophy, periventricular leukomalacia, corpus callosum } \\
\text { anomalies, Dandy-Walker syndrome, Joubert syndrome, cyctic dilataion of 4th ventricle, } \\
\text { polymicrogyria, right hemispheric cyst, partial empty sella, ventriculomegally, encephalomalacia, } \\
\text { watershed infarcts. }\end{array}$ \\
\hline Acquired disorders & $6(5)$ & $\begin{array}{l}\text { Encephalomalacia, acquired hydrocephalus, cortical contusion, hydrocephalus with right } \\
\text { ventricular shunt. }\end{array}$ \\
\hline No data available & $21(19)$ & \\
\hline Total & $115(100)$ & \\
\hline
\end{tabular}

Values are presented as number (\%).

MRI, magnetic resonance imaging; alED, atypical interictal epileptiform discharge. 
longer the time course of the EPSPS, resulting in a longer duration of IEDs (exemplified by SSWs). Second, the frequency, phase, and amplitude of the waveforms combine to generate various types of IEDs (e.g., sharp waves, spikes, spike-wave complexes). Third, spatio-temporal averaging of electrical signals is generated in the cortex when projecting to the scalp. When these projections are widespread the discharges become asynchronous, reaching the scalp with different latencies, resulting in increased duration and blunted waveforms (typified by SSW). Conversely, sharply localized phenomena, such as PSWs, are scarcely recordable from the scalp. Fourth, the synchrony of firing in intracranial recordings determines the amplitude of IEDs. Therefore, the greater the synchronicity, the larger the size of single waveforms.

In the present study, we have presented data on a subgroup of IEDs ("alEDs") that have distinct characteristics. To explain this finding, it is important to discuss the pathophysiological mechanisms of this subgroup and the several theories that have been posited. PSWs, which have systematically been studied in the past, ${ }^{10-14}$ are believed to be induced by cortical deafferentation leading to augmentation of the deep cortical EPSPs. This induces a prominent "positive trough", which is recorded as PSWs, obfuscating the negative component of the spike or sharp wave. Similarly, cortical deafferentation has been implicated in the genesis of FTSWs, where pacemakers located in deeper structures of the brain, such as the entorhinal-hippocampus complex may be the source of this activity. ${ }^{15}$ SSWs are thought to originate in lower cortical layers where augmentation of deep cortical EPSPs occurs. ${ }^{16}$ This is followed by widespread projection of this activity to the surface, inducing SSWs. Theoretically, this process is triggered by cortical deafferentation due to a sub-cortical pathology. Bifid spikes ${ }^{17}$ and "notched" delta ${ }^{18}$ are presumed to emanate from conduction defects in projection fibers, resulting in the electrical signals reaching the scalp with different latencies, configuring a "notched" appearance.

At this juncture, we wish to posit an alternate theory for the generation of alEDs as follows: it is conceivable that some of these patients may have a co-existing type 1 focal cortical dysplasia (FCD) that MR images are not able to reveal. ${ }^{19}$ The underlying pathology of FCD is either cortical laminar disruption or cortical architectural anomaly, which could affect the polarity or morphology of IEDs. Clinically, patients with type 1 FCD present with early or, more typically, adult-onset temporal lobe epilepsy, which is often associated with mental retardation. The typical EEG finding in FCD is temporal lobe IEDs, whereas stereotyped rhythmic discharges and "ripples" may also be present. ${ }^{20}$ The topography of IEDs (predominantly tem- poral) and the presence of intellectual delay in most of our patients supports this theory. FCD may be genetic or acquired in origin. We anticipate that with further advances in MRI technology, perhaps more cases of type 1 FCD will be discovered, in which case the EEG correlates of this pathology will be further defined.

It is important to note that SSWs, PSWs, and bifid spikes display a narrow field distribution with a limited projection both ipsilaterally and contralaterally, whereas FTSWs have limited projection contralaterally. We believe this limited field distribution reflects the preserved integrity of the "inhibitory restraint" (also known as "surround inhibition") that interferes with regional or transcallosal transmission.

The high incidence of congenital CNS anomalies in the alED group (39\%) versus the IED group (6\%) is in accord with previous findings published in the literature. ${ }^{14-18}$ For example, FTSW are characteristic of Rolandic epilepsy whereas ND was originally thought to be pathognomonic of Angelman syndrome. Additional broad-based studies of alED in the future may reveal associations between these EEG patterns and certain congenital CNS anomalies enabling the alEDs to serve as biomarkers for these disorders.

We found a higher incidence of alEDs in the younger age groups, primarily affecting those in the first two decades of life. We posit three theories to explain this demographic preference. First, with the passage of time, there is an evolution of alEDs caused by a decline in the ability of the ageing cortex to generate these discharges. Second, chronic anticonvulsant therapy may suppress this activity. Finally, the high risk of early death in the group with a congenital anomaly, which constituted a significant number of our cohorts, precludes any longterm EEG analysis.

The prevalence of individual alEDs in our sample (1,250 patients) was very low $(9.2 \%)$. However, since the study was conducted at a tertiary-care facility, there could be a referral bias in the recruitment of patients. Therefore, it is difficult to determine the prevalence of these patterns within the general population.

In conclusion, alEDs are rare and under-reported EEG patterns with focal and potential epileptogenic significance. Although several mechanisms may be involved in the pathogenesis of alEDs, we believe the most compelling theory to be the existence of deep cortical pacemakers. Further, the presence of alEDs may indicate co-existing chronic or static central nervous system pathologies, predominantly congenital central nervous system anomalies. Thus, our study the significance of neuroimaging (particularly MRI) in investigating patients with alEDs. Future research should focus on the evaluation of alEDs 
in individual cases, and on applying more sophisticated neuroimaging technology to further define the underlying pathophysiological mechanisms of these patterns.

\section{References}

1. Goldensohn ES, Purpura DP. Intracellular potentials of cortical neurons during focal epileptogenic discharges. Science 1963;139:840-2.

2. Daub JR, Stead SM. Basics of neurophysiology. In: Daube JR, Rubin DI, eds. Textbook of clinical neurophysiology. 3rd ed, Oxford: Oxford University Press, 2009;69-96.

3. Brazier MAB, Petche $H$. Synchronization of EEG activity in epilepsies. New York: Springer, 1972;431.

4. A glossary of terms most commonly used by dinical electroencephalographers. Electroencephalogr Clin Neurophysiol 1974;37:538-48.

5. Ebersole JS. Defining epileptogenic foci: past, present, future. J Clin Neurophysiol 1997;14):470-83.

6. American Clinical Neurophysiology Society. Guideline 8: guidelines for recording clinical EEG and digital media. J Clin Neurophysiol 2006;23: 122-4.

7. Maulsby RL. Some guidelines for assessment of spikes and sharp waves in EEG tracings. Am J EEG Technol 1971;11:3-16.

8. Scheffer IE, Berkovic S, Capovilla G, et al. ILAE classification of the epilepsies: position paper of the ILAE commission for classification and terminology. Epilepsia 2017;58:512-21.

9. Hashiguchi K, Morioka T, Yoshida F, et al. Correlation between scalp-recorded electroencephalographic and electrocorticographic activities dur- ing ictal period. Seizures 2007;16:238-47.

10. Matsuo F, Knott JR. Focal positive spikes in electroencephalography. Electroencephalogr Clin Neurophysiol 1977;42:15-25.

11. Lombroso CT. Neonatal polygraphy in full-term and premature infants: a review of normal and abnormal findings. J Clin Neurophsio/ 1985; 2:105-55.

12. Nowack WJ, Janati A, Antuago T. Positive temporal sharp waves in neonatal EEG. Clin Electroencephalogr 1989;20:196-201.

13. Nowack WJ, Janati A. Positive sharp waves in neonatal EEG. Am J EEG Technol 1990:30:211-21.

14. Janati $A B$, Umair $M$, Alghasab NS, Al-Shurtan KS. Positive sharp waves in the EEG of children and adults. Neurol Sci 2014;35:707-13.

15. Janati $A B, A l G h a s a b N$, Umair $M$. Focal triphasic sharp waves and spikes in the electroencephalogram. Neurol Sci 2015;36:221-6.

16. Janati $A B$, Alghasab NS, Alshammari RA, saad AlGhassab A, Al-Aslami Yossef Fahad. Sharp slow waves in the EEG. Neurodiagn J 2016;56: 83-94.

17. Janati $A B, A l G h a s a b N S$, Aziz $T$, et al. EEG spikes resembling cardiac M-shaped waves in the EKG: the cerebral M pattern. Ann Clin Neurophysiol 2017; 19:58-63.

18. Janati $A B$, Alghasab NS, Abdelwahed $T$, et al. Sharp slow waves in the EEG. Neurodiagn J 2016;56:83-94.

19. Kabat J, Król P. Focal cortical dysplasia - review. Pol J Radio/ 2012;77: 35-43.

20. Seifer G, Blenkmann A, Princich JP, et al. Noninvasive approach to focal cortical dystrophy: clinical, EEG and neuro imaging features. Epilepsy Res Treat 2012;2012:736784. 